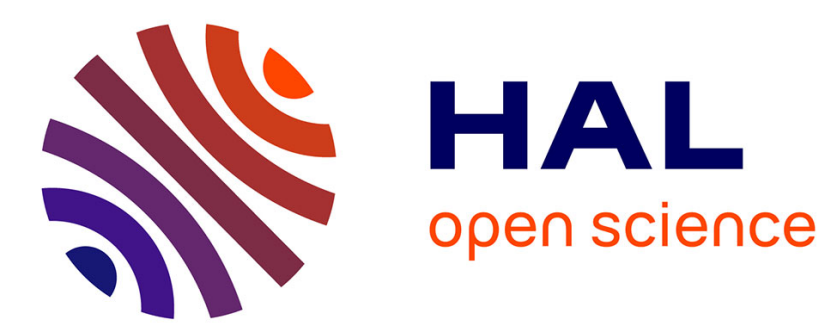

\title{
THE DEFECT STRUCTURE OF COMPOUNDS WITH THE RUTILE STRUCTURE
}

\author{
C. Catlow, R. James, M. Norgett
}

\section{To cite this version:}

C. Catlow, R. James, M. Norgett. THE DEFECT STRUCTURE OF COMPOUNDS WITH THE RUTILE STRUCTURE. Journal de Physique Colloques, 1976, 37 (C7), pp.C7-443-C7-448. 10.1051/jphyscol:1976799 . jpa-00216959

\section{HAL Id: jpa-00216959 https://hal.science/jpa-00216959}

Submitted on 1 Jan 1976

HAL is a multi-disciplinary open access archive for the deposit and dissemination of scientific research documents, whether they are published or not. The documents may come from teaching and research institutions in France or abroad, or from public or private research centers.
L'archive ouverte pluridisciplinaire HAL, est destinée au dépôt et à la diffusion de documents scientifiques de niveau recherche, publiés ou non, émanant des établissements d'enseignement et de recherche français ou étrangers, des laboratoires publics ou privés. 


\title{
THE DEFECT STRUCTURE OF COMPOUNDS WITH THE RUTILE STRUCTURE
}

\author{
C. R. A. CATLOW $(*)$, R. JAMES $(\dagger)$ and M. J. NORGETT \\ Theoretical Physics Division, AERE, Harwell, U. K.
}

\begin{abstract}
Résumé. - Nous présentons une étude théorique sur les propriétés de défauts dans trois composés, $\mathrm{MnF}_{2}, \mathrm{MgF}_{2}$ et $\mathrm{TiO}_{2}$. Nos résultats sont en contraste avec la structure de défauts connue pour les fluorites. Nous trouvons que les désordres du type Schottky sont intrinsèques dans tous les trois composés, avec le transport des ions dû à la mobilité des lacunes dans le cas pur ou dopé. Les études antérieures sur les rutiles d'oxyde et de fluoride avaient plutôt suggérée des désordres du type interstitiel. Nous montrons cependant que notre proposition sur la structure de défaut est compatible avec les récents résultats de Park et Nowick [2] sur la conductivité dans $\mathrm{MgF}_{2}$ et $\mathrm{MnF}_{2}$. Nous présentons ensuite une étude détaillée sur la migration des lacunes qui montre, pour les deux types de lacunes, que le mécanisme dominant conduit à une conductivité largement isotropique. Ce résultat est en accord avec l'expérience faite sur les cristaux dopés d'ions trivalents. Avec des cristaux dopés d'ions mono-valents on a observé une forte anisotropie, ce qui est peut-être dû à la mobilité des ions dopés.
\end{abstract}

\begin{abstract}
We present a theoretical study of the defect properties of three compounds, $\mathrm{MnF}_{2}$, $\mathrm{MgF}_{2}$ and $\mathrm{TiO}_{2}$. Our results predict a defect structure which is in marked contrast to that characteristic of fluorite compounds. We propose that Schottky disorder is intrinsic to all three crystals, with ion transport effected by vacancy mobility in both pure and doped materials. Earlier studies on both fluoride and oxide rutiles have, however, suggested interstitial disorder. Nevertheless, we show that our proposals are compatible with the recent data of Park and Nowick [2] on the conductivity of $\mathrm{MgF}_{2}$ and $\mathrm{MnF}_{2}$. We then report a detailed study of vacancy migration and we find that, for both anion and cation vacancies, the dominant mechanism is one which will result in largely isotropic conductivity. This accords with the experimental data on crystals doped with trivalent ions ; but for monovalent doped crystals, high anisotropy has been observed, which may, however, be due to dopant mobility.
\end{abstract}

1. Introduction. - The defect properties of compounds with the rutile structure (e. g. $\mathrm{MnF}_{2}, \mathrm{TiO}_{2}$ ) have been far less extensively studied than those of corresponding fluorite crystals (e. g. $\mathrm{CaF}_{2}, \mathrm{UO}_{2}[1]$ ). The rutiles are more complex systems, and in particular their non-cubic structure, illustrated in figure 1 , leads to anisotropy in defect properties - a problem not encountered for the cubic fluorites. In this paper, we examine the defect structure and transport properties of rutiles using theoretical methods which we have applied with considerable success to the fluorites $[6,7$, 11]. Our results suggest that there are fundamental differences between the two types of compound, both in the nature of the intrinsic disorder and in the defect transport mechanisms. We concentrate mainly on the rutile fluorides, $\mathrm{MnF}_{2}$ and $\mathrm{MgF}_{2}$ for which some experimental data are available [2], but the more complex problems presented by the oxide, $\mathrm{TiO}_{2}$ are briefly discussed.

$\left.{ }^{*}\right)$ Present address : Dept. of Chemistry, University College, Gower Street, London, WCl.

(f) On attachment from : Inorganic Chemistry Laboratory, Oxford.

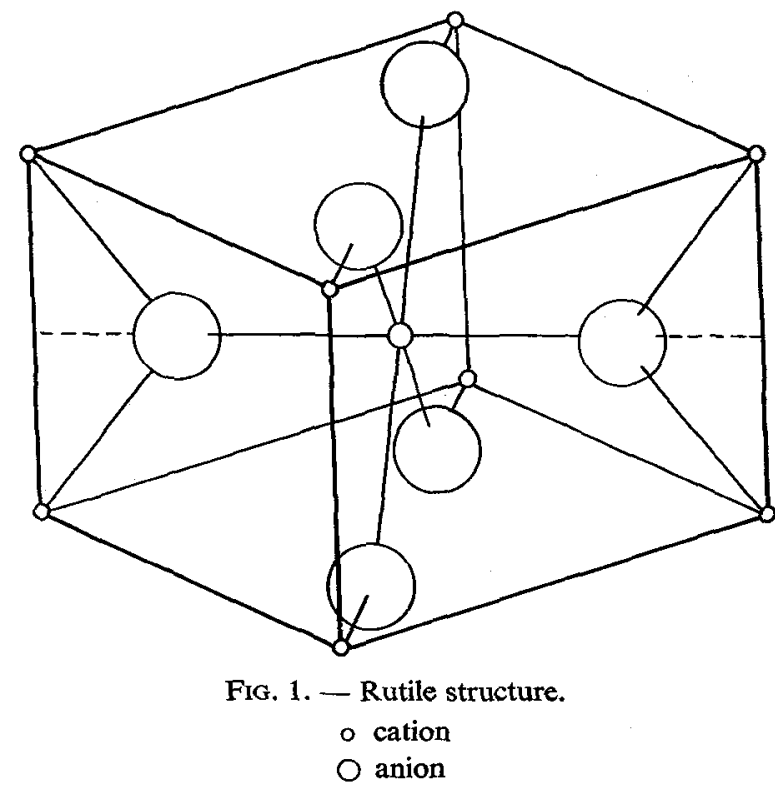

In the next section, we give a brief account of our calculations. Our results are then presented in section 3 and discussed in section 4 in the light of the experimen- 
tal data of Park and Nowick [2]. We conclude with a summary of our main proposals, and some suggestions for further study.

2. Calculations and lattice models. - Our calculation of defect structures and energetics use the HADES program. This is a general package for defect studies on ionic crystals which is discussed elsewhere in this conference [5] and for which other more detailed accounts are available $[3,4]$. A number of studies have clearly shown that the methods used by Hades are reliable $[3,5,6,7]$. Uncertainties in the calculated defect energies reflect only uncertainties in the potentials used to describe lattice and defect-lattice interactions. In the remainder of this section therefore we concentrate on the problem of potentials.

Following our earlier study of fluorite oxides [11] and fluorides [6,7], our lattice potentials are based on the ionic model - that is, we simulate the crystals by entities of integral charge. Short range interionic interactions are then described using simple analytical potentials, of Born-Mayer or Buckingham form $[5,6$, $7,8]$; ionic polarisation is treated using the shell model whose advantages for defect studies are discussed in a number of studies $[3,6]$, and elsewhere in this conference [5].

In deriving parameters for potentials used in defect studies, we have generally used empirical methods [6,7] : variable parameters of our lattice model have been fitted to experimental dielectric and elastic data and to equilibrium structural properties (which for simple cubic crystals such as the alkali halides and alkaline earth fluorides comprise only the lattice parameter). We use the same approach in our derivation of potentials for the rutile compounds. But this problem is much more complex than that encountered in previous studies of alkali halides $[8,9]$ or fluorites $[6,7]$. This is first because of the anisotropy of the dielectric properties (particularly the very large values of the dielectric constants observed for $\mathrm{TiO}_{2}$ ) but, secondly, because of the greater number of structural parameters to which we must fit our model. In addition to the two lattice parameters for the orthorhombic rutile structure, our model must also reproduce the correct atomic coordinates within the unit cell. These are not entirely determined by symmetry as with simpler structures (e. g. alkali halides and fluorites).

To deal with the complexity of empirical fitting procedures of potentials for low symmetry structures, we have developed a powerful general program (PLUTO) ${ }^{1}$ ). This may be used to fit parameters to both elastic and dielectric crystal data, with the constraint that the potential reproduces the observed structural data - that is both lattice parameters and atomic coordinates within the unit cell. PLUTO was therefore used in the empirical fits in this study, and for

(1) Perfect Lattice Unrestricted Testing Operation. further details of the program we refer to Catlow and Norgett [10].

In deriving an empirical potential for any ionic crystal, a major problem concerns the treatment of second-neighbour potentials $[5,6,7,8]$; in rutiles, as with fluorites, these are anion-anion interactions. Reliable empirical values cannot be derived for shortrange repulsive terms of such potentials, although an accurate description of this interaction is necessary if we are to calculate reliable energies of anion interstitials. In previous studies therefore, we have determined parameters for these interactions using theoretical methods - either with molecular-orbital Hartree-Fock techniques [12] or the simpler electron-gas methods of Wedepohl [13] and Gordon and Kim [14]. We continue this procedure in our study of rutiles.

As a general solution of the problems of describing second-neighbour interactions, we have recently proposed that single analytical forms should not be used over all internuclear separations : rather that we should use separate analytical functions in attractive and repulsive regions of the potential ; these should then be joined by polynomials. More details of this procedure, which we used in one of our potentials for $\mathrm{MnF}_{2}$, are given by Catlow et al. $[5,7,8]$ and James [15].

We have therefore used the PLUTO program to derive empirical potentials for $\mathrm{MnF}_{2}, \mathrm{MgF}_{2}$ and $\mathrm{TiO}_{2}$. The models reproduce elastic, dielectric and structural data. In each case we parameterised a simple model based on Born-Mayer and Buckingham potentials. However for $\mathrm{MnF}_{2}$, in addition to this model (potential 1), we derived a second model (potential 2) in which we used the more flexible spline potential for the second-neighbour interaction. For most defects, calculations using potential 1 should be satisfactory, although the model gives a less accurate fit to the equilibrium structural data. We of course report results for both potentials where possible, as a comparison of results using different models is particularly valuable.

For details of our potential parameters and for more discussion of their derivation we refer to James [15] (who reports several further potentials for $\mathrm{MnF}_{2}$, of which only the first two are used here). The parameters show only one unusual feature : large cation polarisabilities are derived, particularly for $\mathrm{TiO}_{2}$, where we obtained a value of $4.7 \AA^{3}$ for the polarisability of $\mathrm{Ti}^{4+}$. It is difficult to justify ionic polarisabilities of this magnitude, and their derivation, we believe, may indicate serious inadequacies in purely intraionic models of high frequency lattice polarisabilities.

For lattice-defect potentials we followed the procedure of our previous studies. For intrinsic defects, e. g. self interstitials, we used the parameters from the appropriate lattice potential. The extrinsic defects considered were dopant cations ; here we used the same parameters as for the lattice cation, except for the different charge of the dopant ion. This procedure has been used elsewhere $[16,17]$ with satisfactory results. 
TABLE I

Defect formation energies in rutile compounds

\begin{tabular}{ccccc}
\multicolumn{1}{c}{ Crystal } & $\begin{array}{c}\text { Anion } \\
\text { vacancy }\end{array}$ & $\begin{array}{c}\text { Anion } \\
\text { interstitial }\end{array}$ & $\begin{array}{c}\text { Defect energy } \\
\text { Cacancy }\end{array}$ & $\begin{array}{c}\text { Cation } \\
\text { interstitial }\end{array}$ \\
$\mathrm{MgF}_{2}$ & $\overline{-}$ & $-\overline{0}$ & - & - \\
$\mathrm{MnF}_{2}$ potential 1 & 5.67 & -2.02 & 26.54 & -19.05 \\
$\mathrm{MnF}_{2}$ potential 2 & 5.90 & - & 25.11 & -19.56 \\
$\mathrm{TiO}_{2}$ & 5.40 & -6.82 & 22.82 & - \\
& 15.83 & & 78.51 & -69.93
\end{tabular}

3. Results. - In table I we present results of vacancy and interstitial formation energies for the three crystals, which we than combined to give the Schottky and Frenkel energies given in table II. For the interstitial in the rutile structure there are two possibilities - the octahedral site illustrated in figure 2 , and the tetrahedral site. We confirmed that the smaller size of this latter site results in much higher energies than for octahedral interstitials to which our results in table I refer.

\section{TABLE II}

Schottky and Frenkel energies

\begin{tabular}{lccc} 
& \multicolumn{3}{c}{ Defect energy (eV) } \\
\multicolumn{1}{c}{ Crystal } & $\begin{array}{c}\text { Anion } \\
\text { Frenkel } \\
\text { pair }\end{array}$ & $\begin{array}{c}\text { Cation } \\
\text { Frenkel } \\
\text { pair }\end{array}$ & $\begin{array}{c}\text { Schottky } \\
\text { trio }\end{array}$ \\
$\mathrm{MgF}_{2}$ & - & - & - \\
$\mathrm{MnF}_{2}$ potential 1 & 5.09 & 7.49 & 2.18 \\
$\mathrm{MnF}_{2}$ potential 2 & 3.88 & 5.55 & 1.82 \\
$\mathrm{TiO}_{2}$ & - & - & 1.53 \\
& 9.00 & 8.58 & 0.26
\end{tabular}

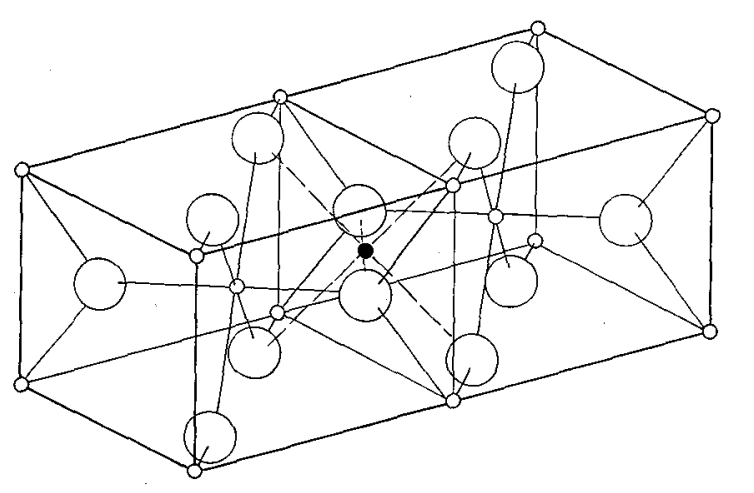

FIG. 2. - Cation interstitial site.

- octahedral site

We present results where possible for both the potentials derived for $\mathrm{MnF}_{2}$. In some cases, however, the calculation failed to converge when we used potential 2. We encountered the same problem in our study of $\mathrm{TiO}_{2}$, where all defects calculations were susceptible to divergence. The results reported for this crystal refer therefore to defect and lattice configurations that are only local energy minima; and the configurations for which divergence occurs are generally close to these minima. This difficulty, which is discussed in greater detail by James [15], must reduce the reliability of our results for $\mathrm{TiO}_{2}$.

As discussed in the following section, our calculated energies indicate Schottky disorder as intrinsic to the crystal; thus the defect structure in both intrinsic and doped crystals will be dominated by vacancies. We therefore continue with calculations on vacancy activation energies. For the anion vacancy there are three migration mechanisms, as illustrated in figure $3:$ the $W_{1}$

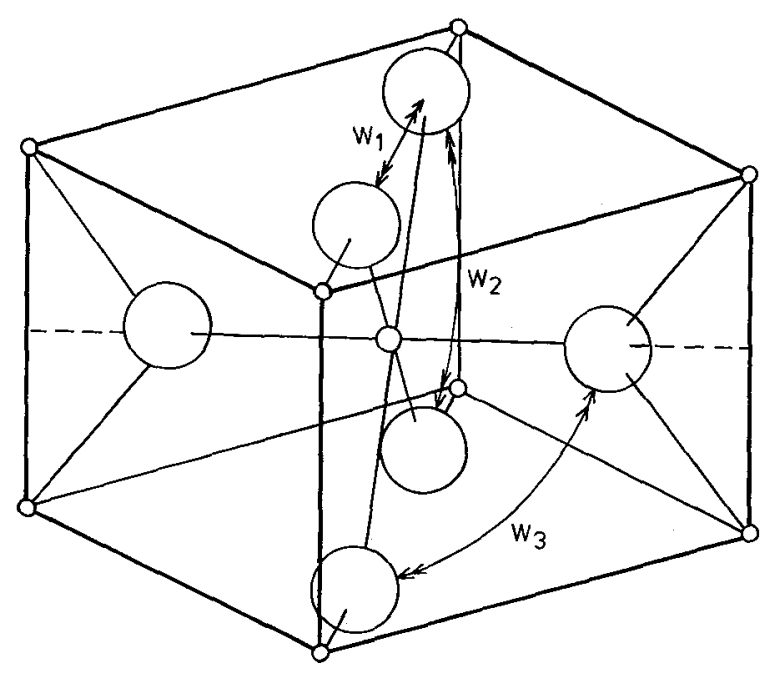

FIG. 3. - Anion vacancy diffusion paths

jump shown in the figure effects transport in the $x y$ plane ; the $W_{2}$ jump is in the $z$ direction ; only the $W_{3}$ jump results in transport both parallel and perpendicular to the $z$ axis - a point of importance to which we return later. The four possible mechanisms for cation vacancy migration are shown in figure 4 : again there are jumps, $V_{1}$ and $V_{3}$, confined to the $x y$ plane, while the $V_{2}$ jump is along the $z$ axis ; and again only one jump, the $V_{4}$, allows both parallel and perpen- 


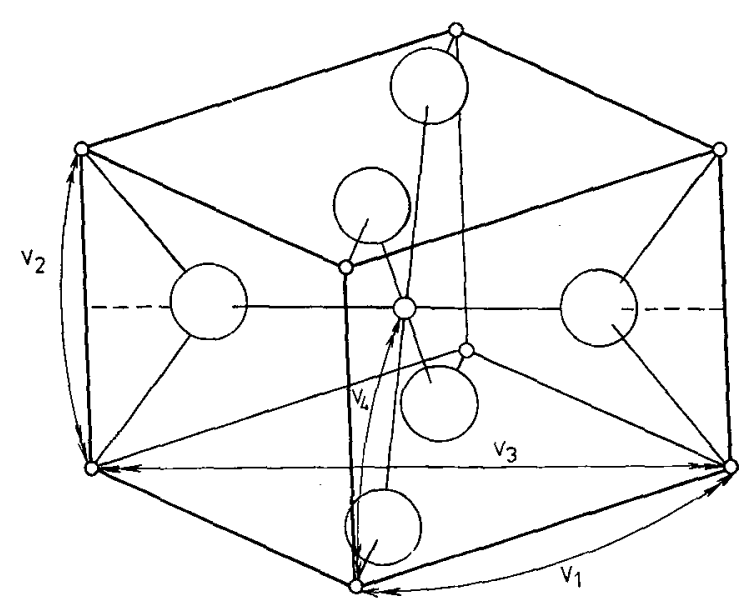

FIG. 4. - Cation vacancy diffusion paths.

dicular migration. We report results for each mechanism for both vacancies in table III. In every case we assumed that the saddle point has the migrating ion at the midpoint of the line joining the two lattice sites between which the vacancy jump occurs.

\section{TABLE III}

\section{Vacancy activation energies}

(a) Anion vacancy.

\begin{tabular}{lccc} 
& & \multicolumn{3}{c}{ Activation energy $(\mathrm{eV})$} \\
Crystal & $W_{1}$ jump & $W_{2}$ jump & $W_{3}$ jump \\
$\mathrm{MgF}_{2}$ & - & - & - \\
$\mathrm{MnF}_{2}$ potential 1 & 0.31 & 1.53 & 0.03 \\
$\mathrm{MnF}_{2}$ potential 2 & -0.44 & 0.90 & 0.04 \\
& 0.61 & 1.23 & -
\end{tabular}

(b) Cation vacancy.

\begin{tabular}{ccccc}
\multicolumn{1}{c}{ Crystal } & \multicolumn{5}{c}{ Activation energy (eV) } \\
\cline { 4 - 5 } & $V_{1}$ jump & $V_{2}$ jump & $V_{3}$ jump & $V_{4}$ jump \\
$\mathrm{MgF}_{2}$ & - & - & - & - \\
$\mathrm{MnF}_{2}$ potential 1 & 0.96 & 1.07 & 10.16 & 0.06 \\
& 0.87 & 1.57 & 8.36 & 1.82
\end{tabular}

Results on vacancy mobility are unfortunately available only for the fluoride crystals, as for $\mathrm{TiO}_{2}$ our calculations failed to converge. A further problem with most of the calculations follows from the low symmetry of the saddle point structure. This restricts the size of the explicitly simulated region of crystal that may be used in the calculation (for further discussion see references [3] and [5]). The accuracy of the results is therefore somewhat reduced.

Finally we consider the clustering between vacancies and dopant ions in the fluoride crystals. Both monovalent and trivalent ions have been introduced into rutile fluorides [2]. Our models, based on vacancy disorder, predict that the cation substitutional dopants will be compensated in the former case by anion vacancies and in the latter by cation vacancies. We therefore considered the following aggregates : first, a cluster of an anion vacancy with a monovalant dopant ion in the nearest neighbour (n. n.) cation site ; secondly clusters of a cation vacancy with one or two trivalent ions in the n. $n$. cation site (only the latter defect is electrically neutral). With $\mathrm{MnF}_{2}$, however, for these two latter clusters containing cation vacancies we can report no results, as our calculations again failed to converge.

We should note that our calculations for dopant ions all neglect effects arising from the differing ion sizes of dopant and host lattice cation, as we made no allowance for any differences in short range potentials between dopant and lattice cation interaction (see previous section). Both such effects should be small compared with the dominant effect due to the electrostatic perturbation of the lattice by the effective charge of the defect. The results for these calculations on dopant-vacancy clusters are presented in table IV.

4. Discussion. - 4.1 Rutile FLuORIDES. - Our most significant result is the prediction of Schottky disorder in rutile fluorides (see Table II). This contrasts with the Frenkel defect behaviour of the fiuorites [1] and is a consequence of the much smaller interstitial site in the rutile structure. As a consequence of the Schottky defect structure, aliovalant cations are compensated by vacancy defects, not by interstitials as in the fluorites.

This conclusion contradicts the opinion of Park and Nowick [2] who deduced Frenkel defect behaviour from their study of conductivity and dielectric relaxation in pure and doped $\mathrm{MnF}_{2}$. There is indeed strong if indirect evidence for anion vacancy defects in mono-

TABLE IV

Vacancy-Dopant interaction energies

\begin{tabular}{|c|c|c|c|}
\hline \multirow[b]{2}{*}{ Crystal } & \multicolumn{3}{|c|}{ Defect interaction energy $(\mathrm{eV})\left(^{*}\right)$} \\
\hline & $\begin{array}{l}\text { Anion vacancy- } \\
\text { monovalent dopant } \\
\text { pair }\end{array}$ & $\begin{array}{c}\text { Cation vacancy- } \\
\text { trivalent dopant } \\
\text { pair }\end{array}$ & $\begin{array}{l}\text { Trio of one cation } \\
\text { vacancy plus two } \\
\text { trivalent dopants }\end{array}$ \\
\hline- & - & - & - \\
\hline & -0.56 & -1.35 & -2.63 \\
\hline otential 1) & -0.48 & - & - \\
\hline
\end{tabular}

$\mathrm{MgF}_{2}$ (potential 1)

$-0.48$

(*) All clusters have dopant ions $\mathbf{n}$. $n$. with respect to vacancies. All energies are given with respect to isolated component defects. 
valent doped $\mathrm{MnF}_{2}$. Such models accord best with the observed anisotropy in conductivity and permit a rational analysis of the results of dielectric relaxation studies. But the detection of anion vacancies in such crystals in compatible with either Schottky or anionFrenkel models. It eliminates only cation-Frenkel behaviour which has been proposed in earlier work in $\mathrm{MgF}_{2}$ [18] but which our calculations eliminate.

Park and Nowick discuss indirect evidence for the presence of anion interstitials in trivalent doped crystals which, if confirmed, would establish the anionFrenkel model. The evidence for this behaviour rests first on the absence from the dielectric relaxation results of a peak characteristic of a cation vacancy-dopant complex. But equally they find no dopant-interstitial complex to support their proposed Frenkel model. In either case, the difficulty in obtaining satisfactory assignments may be due to more extensive defect clustering.

The more substantial argument for the anionFrenkel model is the observed isotropy of the conductivity of trivalent-ion-doped crystals. This seems more reasonable for anion-interstitial transport than for cation-vacancy conduction. Park and Nowick argue that in this latter case, the anisotropy should be large because the $V_{2}$ cation-vacancy jump (see Fig. 4) should have a substantially lower activation energy than any alternative mechanism. This contributes only to $\sigma_{1}$, the conduction in the $z$ direction.

Our results (Table III $b$ ) do not justify this argument. There is no simple correlation between jump length and activation energy as postulated by Park and Nowick. In $\mathrm{MgF}_{2}$, we find that the $V_{4}$ jump dominates with a low activation energy giving isotropic conduction. In $\mathrm{MnF}_{2}$, the $V_{1}$ jump is preferred which contributes only to $\sigma_{\perp}$ in the $x y$ plane.

The different behaviour of $\mathrm{MgF}_{2}$ and $\mathrm{MnF}_{2}$ indicates that the relative activation energies for the different cation jumps depend sensitively on the assumed interionic potential. This must adversely affect the quantitative value of our results. More importantly, our results depend on the assumption that the moving cation passes directly from one site to the other through a saddle-point midway between the two sites. This is a much more reasonable approximation for the $V_{1}$ and $V_{2}$ transitions where the direct path is largely unimpeded. For the $V_{4}$ jump, a curved path may well locate a more favourable saddle-point. This will mean that the actual activation energy falls below our estimate made using a direct path. If, as we believe, we have overestimated the energy of the $V_{4}$ jump for $\mathrm{MnF}_{2}$ and the error is larger than for the $V_{1}$ and $V_{2}$ jumps, then we can reconcile isotropic conductivity by the $V_{4}$ mechanism with Schortky defect behaviour in these crystals.

In returning now to discuss conduction in monovalent doped materials, with anion vacancy excess, the problem now is to explain the observed anisotropic conduction. Again, the most striking feature of our results for anion-vacancy migration is the sensitivity of the results to the details of the potential. In particular, for the $W_{1}$ jump, the two potentials for $\mathrm{MnF}_{2}$ give differences in activation energy of $\sim 1 \mathrm{eV}$ (Table III $a$ ); indeed potential 1 predicts a negative activation energy corresponding to a split vacancy structure. It is fortunate that the $W_{1}$ jump is relatively unimportant as it can make no contribution to conductivity. The two lattice sites linked form a particular close pair. The $W_{1}$ jump might, however, contribute in relaxation experiments.

Our results predict that anion-vacancy migration will be effected by the $W_{3}$ jump, for which very low activation energies are calculated. This mechanism would lead to isotropic conductivity, as discussed in the previous section, in contrast to the experimental observation of anisotropy. The conductivity ratio $r\left(=\sigma_{\|} / \sigma_{\perp}\right)$ increases with dopant concentration to a value of $\sim 460$ for $1000 \mathrm{ppm}$ of $\mathrm{Li}^{+}$doped in $\mathrm{MnF}_{2}$. To explain their results Park and Nowick [2] propose that the activation of energies for the $W_{1}$ and $W_{3}$ jumps are very similar - a fortuitous coincidence which is not supported by our calculations. An alternative possibility is that the $\mathrm{Li}^{+}$used as dopant is itself mobile, making a major contribution to the conductivity. We will shortly report a theoretical study of $\mathrm{Li}^{+}$ migration in $\mathrm{MnF}_{2}$ and $\mathrm{MgF}_{2}$; and clearly further work, both experimental and theoretical remains to be done on the problem of the conductivity anisotropy in monovalent doped rutile fluorides.

A detailed discussion of vacancy-dopant interaction, for which we present results in table IV, is clearly not possible, both because of the failure of some of our calculations on $\mathrm{MnF}_{2}$ and because of the paucity of experimental data. Quantitative comparison is, however, possible between the results of our calculation on monovalant dopant-vacancy interactions and the values deduced from the conductivity data of Park and Nowick [2]. We calculate 0.48 and $0.56 \mathrm{eV}$ for the dopant-vacancy binding energy in $\mathrm{MnF}_{2}$ and $\mathrm{MgF}_{2}$ respectively; the experimental values are 0.73 and $0.82 \mathrm{eV}$. The discrepancy, if significant, may reffect the existence of more extensive clustering in the doped samples than was assumed in our calculation. Regarding the trivalent doped crystals, our calculations predict that both charged vacancy-dopant pair and the neutral complex of one vacancy and two dopant ions will be important. For if we consider the following disproportionation reaction :

$$
2\left[\mathrm{M}^{3+} \mathrm{V}^{2+}\right] \rightarrow \mathrm{V}^{2+}+\left[\mathrm{M}_{2}^{3+} \mathrm{V}^{2+}\right]
$$

(where by $\left[\mathrm{M}^{3+} \mathrm{V}^{2+}\right]$ we mean the pair complex, by $\mathrm{V}^{2+}$, an isolated cation vacancy, and by $\left[\mathrm{M}_{2}^{3+} \mathrm{V}^{2+}\right]$ the trio complex), we calculate, using the results in table IV, an energy of reaction of $0.06 \mathrm{eV}$. This will lead to an equilibrium constant of order unity. The possibility of the co-existence of these clusters should therefore be considered in discussions of dielectric relaxation in trivalent doped rutiles. 
4.2 $\mathrm{TiO}_{2} .-$ We give only a brief discussion of our results here, as we believe that the uncertainties in our calculated energies are greater than for the fluoride crystals. As commented, our results on $\mathrm{TiO}_{2}$ all refer to local minima, close to which are regions in which the defect energy diverged ; the reliability of the results is then clearly reduced. However, one clear feature emergies from calculations, that is the dominance of Schottky disorder in line with our prediction for the rutile fluorides. And although our calculated Schottky energy is evidently too low, the difference between calculated Schottky and Frenkel energies is nevertheless large and outside the probable range of uncertainty in our calculations. We predict therefore that in slightly reduced rutile, $\mathrm{TiO}_{2-x}$, oxygen vacancies will be present, as suggested by Iguchi and Yajima [19], but that interstitials as proposed in a number of studies (see e. g. Kofstad [20]) will be unimportant. The problem of the defect structure of $\mathrm{TiO}_{2-x}$ is complicated by the nucleation of extended defects (see e. $\mathrm{g}$. Bursill and Hyde [21]) at all but very low values of $x$. However, in the region where point defects are present, our results clearly show that vacancy disorder will predominate. A more detailed discussion of point defects in $\mathrm{TiO}_{2-x}$ is given by James [15].

5. Conclusions. - It is quite clear that the reliability of theoretical estimates of defect energies in rutile compounds is at present less than that which we have achieved for corresponding fluorite crystals $[6,7,11]$; our conclusions must therefore be tentative. But certain definite predictions follow from our study in the paper - principally the proposal of Schottky disorder which we have shown is compatible with the experimental transport data, but which is at present unconfirmed. The detection of cation vacancies in crystals doped with trivalent cations would verify our proposal; and we have therefore undertaken a diffraction study of $\mathrm{Sc}^{3+}$ doped $\mathrm{MnF}_{2}$ [15], for which we have however, as yet no conclusive results. Our second proposal concerns vacancy migration for which we have suggested mechanisms - the $W_{3}$ and $V_{4}$ iumps in figures 3 and 4 which would lead to roughly isotropic conductivity. In the case of anion migration, this prediction is difficult to reconcile with the observed anisotropy of the conductivity ; we have suggested dopant mobility as a possible solution. Finally, our work on $\mathrm{TiO}_{2}$, predicts that vacancy disorder will predominate in the crystal as well as in the fluoride compounds, which contrasts with previous suggestions [20]. Our study has therefore suggested that radical differences may exist between the defect structure of rutile and fluorite crystals, and that rutile compounds should provide considerable further interest for experimental and theoretical work.

Acknowledgments. - We are grateful to Dr. B. E. F. Fender for a number of useful discussions.

\section{References}

[1] Crystals with the Fluorite Structure, ed. W. Hayes (Clarendon Press) 1974.

[2] Park, D. S. and Nowick, A. S., J. Phys. \& Chem. Solids 37 (1976) 607 [see also PARK, D. S. Columbia Unit Tech report, Science Foundation Grant No. GH-34269].

[3] Lidiard, A. B. and Norgett, M. J., Computational Solid State Physics eds. W. Herman et al. (Plenum Press, New York) 1972.

[4] NoRgETt, M. J., UKAEA report, AERE-R7650 (1974).

[5] Catlow, C. R. A., Corish, J., Diller, K. M., JACoBs, P. W. M. and Norgetr, M. J., J. Physique Colloq. 37 (1976) C7, this issue.

[6] Catlow, C. R. A. and Norgett, M. J., J. Phys. C. 6 (1973) 1325 .

[7] Catlow, C. R. A., Norgett, M. J. and Ross, T. A., UKAEA Report AERE-TP673 (1976).

[8] Catlow, C. R. A., Diller, K. M., NorgetT, M. J., UKAEA Report AERE-TP672 (1976).

[9] Drllek, K. M., D. Phil. Thesis, University of Oxford, 1976.

[10] Catlow, C. R. A. and Norgett, M. J., UKAEA Report AERE-R8443 (1976).
[11] Catlow, C. R. A. and Lidiard, A. B., Proc. International Symposium, Thermodynamics of Reactor Materials, IAEA, Vienna, 1974, Vol. II, p. 27.

[12] Catlow, C. R. A. and Hayns, M. R., J. Phys. C 5 (1972) L237.

[13] Wedepohl, P. T., Proc. Phys. Soc. 92 (1967) 79.

[14] Gormon, R. G. and KIM, Y. S., J. Chem. Phys. 56 (1972) 3122.

[15] JAMES, R., UKAEA Report-TP666 (1976).

[16] Catlow, C. R. A., J. Phys. C 6 (1973) L64.

[17] Catlow, C. R. A., J. Phys. C 9 (1976) 1845.

[18] Volynets, F. K. and Dronova, G. N., Sov. Phys. Solid. State 11 (1970) 1504.

[19] Iguchi, E. and YajIma, K., J. Phys. Soc. Japan 32 (1972) 1415.

[20] KofSTAD, P., Non Stoichiometry, Diffusion and Electrical Conductivity in Binary Metal Oxides (John Wiley) 1972.

[21] Bursill, L. A. and Hyde, B. G., Prog. Solid State Chem. 7 (1972) 177. 